LUCIANE RIBEIRO ${ }^{1}$

RONALDO ROCHA BASTOS ${ }^{2}$

LUIZ CláUdio Ribeiro ${ }^{2}$

Marcel de Toledo VieirA ${ }^{2}$

Isabel CRistina Gonçalves Leite ${ }^{3}$

Maria Teresa Bustamante TeixeIRA ${ }^{4}$

\title{
Não adesão às diretrizes para rastreamento do câncer do colo do útero entre mulheres que frequentaram o pré-natal
}

\author{
Non adherence to cancer screening guidelines for \\ cervical cancer among women who attended prenatal care
}

Artigo Original

\section{Palavras-chave \\ Neoplasias do colo do útero Esfregaço vagina Saúde da mulher Inquéritos epidemiológicos Análise multivariada \\ Keywords \\ Uterine cervical neoplasms Vaginal smears \\ Women's health \\ Health surveys Multivariate analysis}

\section{Resumo}

OBJETIVO: Estimar a prevalência e identificar fatores associados ao exame citopatológico do colo do útero em atraso (realizado há mais de três anos) entre mães com filhos menores de dois anos de idade que frequentaram o exame prénatal. MÉTODOS: Estudo transversal, de base populacional, com inquérito domiciliar. Os critérios de inclusão foram: mulheres com filhos menores de dois anos, residentes na Zona Norte do município de Juiz de Fora (MG), na data da entrevista. Utilizou-se amostragem complexa com estratificação e conglomeração. Aplicou-se um questionário que abordou questões demográficas e socioeconômicas, além de informações sobre o pré-natal e as práticas de prevenção do câncer do colo do útero. Para análise estatística dos fatores associados, foi utilizado o teste do $\chi^{2}$ e, posteriormente, o modelo de regressão logística com as variáveis que apresentaram nível de significância menor ou igual a 0,05 na análise bivariada. RESULTADOS: Encontrou-se uma prevalência de exame em atraso de 26,6\% (IC95\% $21,3-32,6$ ), incluindo as mulheres que nunca se submeteram ao exame citopatológico (CP) do colo do útero anteriormente. As variáveis com associação significativa à não submissão ao exame no prazo estipulado foram: estado civil casada IOR 0,5; IC95\% 0,2-0,9) e separada/viúva (OR 0, 1; IC95\% 0,02-0,8), ter se submetido ao exame ginecológico no pré-natal $(O R$ 0,3; IC95\% 0, 1-0,6) e número de consultas pré-natal IOR 0,09; IC95\% 0,03-0,25 para mais de 11 consultas), todos fatores de proteção. CONCLUSÕES: A prevalência de exame citopatológico atualizado está ligeiramente abaixo do indicado pela Organização Mundial da Saúde. Além disso, o fato de ter frequentado o prénatal não foi determinante para garantir a realização do exame citopatológico segundo periodicidade recomendada.

\section{Abstract}

PURPOSE: To estimate the prevalence and identify the factors associated with delayed pap smear test of the cervix (carried out more than three years ago) among mothers with sons under two years of age, who attended the prenatal care. METHODS: Cross-sectional, population-based household survey. Women with sons under two years old, living in the northern area of the city of Juiz de Fora (MG), Brazil, were interviewed. Stratification and clustering were used in a complex sampling procedure. We applied a survey questionnaire to capture women's demographic and socioeconomic characteristics of women and information about prenatal and practices for preventing cancer of the cervix. For statistical analysis of the possible bivariate association of factors, we used $\chi^{2}$ test and a logistic regression model with the explanatory variables that had a significance less than or equal to 0.05 in the bivariate analysis. RESULTS: We found a delayed test prevalence of $26.6 \%(95 \% \mathrm{Cl} 21.3-32.6)$, including women who were never submitted to the exam. The variables associated with the non-adherence to the examination within the stipulated time were: to be married (OR 0.5; $95 \% \mathrm{Cl} 0.2-0.9$ ), and divorced/widowed (OR 0.1; 95\% Cl 0.02-0,8), having performed gynecological examination in prenatal care $(O R \quad 0.3 ; 95 \% \mathrm{Cl} 0.1-0.6)$ and number of prenatal visits $(O R 0.09 ; 95 \% \mathrm{Cl} 0.03-0.25$ for more than 11 visits), being all protection factors. CONCLUSIONS: The prevalence of adherence to Pap smear guidelines is slightly lower than the percentage recommended by the World Health Organization. Apart from that, the fact that the woman was submitted to the prenatal care did not guarantee the adherence to Pap smear frequency guidelines.
Correspondêncio

Luciane Ribeiro

Departamento de Saúde Coletiva, Faculdade de Medicino Universidade Federal de Juiz de Forn Rua José Lourenço Kelmer, $s / n$ - Campus Universitório - Martelos CEP: $36036-900$ Juiz de Fora (MG), Brasi

Recebido

$27 / 05 / 2013$

Aceito com modificacōes $31 / 07 / 2013$
Trabalho realizado no Departamento de Saúde Coletiva da Universidade Federal de Juiz de Fora - UFJF - Juiz de Fora (MG), Brasil. 'Departamento de Saúde Coletiva, Faculdade de Medicina, Universidade Federal de Juiz de Fora - UFJF - Juiz de Fora (MG), Brasil. ${ }^{2}$ Departamento de Estatística, Universidade Federal de Juiz de Fora - UFJF - Juiz de Fora (MG), Brasil.

${ }^{3}$ Faculdade de Medicina, Universidade Federal de Juiz de Fora - UFJF - Juiz de Fora (MG), Brasil.

4Programa de Pós-Graduacão em Saúde Coletiva; Núcleo de Assessoria, Treinamento e Estudos em Saúde, Universidade Federal de Juiz de Fora - UFJF - Juiz de Fora (MG), Brasil.

Conflito de interesses: não há. 
Introdução

Dentre os diversos tipos de neoplasias que acometem a mulher, o câncer do colo do útero (CCU) vem ocupando posição de destaque como problema de Saúde Pública, devido à sua alta frequência, principalmente nos países em desenvolvimento ${ }^{1-3}$. No Brasil, para o ano de 2013, são esperados 17.540 casos novos da doença, com risco estimado de 17 casos para cada 100 mil mulheres. Desde 2003, excetuando-se o câncer de pele não melanoma, o CCU se mantém como o segundo tipo de neoplasia mais frequente entre as mulheres, superado apenas pelo câncer de mama ${ }^{4}$.

A frequência elevada dessa neoplasia desperta a atenção, uma vez que possui uma história natural conhecida, possibilitando sua prevenção e detecção precoce e permitindo um excelente prognóstico ${ }^{5}$. No Brasil, desde 1988, o Ministério da Saúde (MS) recomenda a detecção precoce da doença por meio do rastreamento de mulheres sexualmente ativas, utilizando-se o exame citopatológico (CP) do colo do útero, também conhecido como teste de Papanicolaou, considerado como um método efetivo, de baixo custo e de fácil execução ${ }^{6-8}$.

No que se refere ao cuidado à mulher durante o período pré-natal nos serviços de Atenção Primária à Saúde (APS), o MS dispõe de um manual técnico de orientação que deve ser utilizado como referência pelos profissionais de saúde envolvidos nesse tipo de assistência. Considerando-se as diversas orientações contidas nesse manual, o CP do colo do útero deverá ser indicado para todas as gestantes que nunca se submeteram ao exame ou que estão em atraso quanto à sua realização (última coleta há mais de três anos) $)^{9,10}$.

Partindo do princípio de que a procura pelos serviços de saúde para o acompanhamento pré-natal deve ser sempre considerada como uma oportunidade para indicação do rastreamento do $\mathrm{CCU}^{11,12}$, reconhece-se como importante a realização de estudos de base populacional envolvendo mulheres que frequentaram o pré-natal. Essas pesquisas são necessárias para estimar a prevalência de realização do $\mathrm{CP}$ do colo do útero conforme recomendação do MS, bem como avaliar o impacto das ações de saúde direcionadas para o controle do CCU e da assistência ao pré-natal. Além disso, possibilitam a definição de metas a serem alcançadas que permitam um planejamento direcionado às reais necessidades de saúde ${ }^{10,13}$.

Nessa perspectiva, estudos de base populacional têm sido realizados em municípios brasileiros, utilizando-se amostras populacionais diversificadas, com o objetivo de avaliar a cobertura do $\mathrm{CP}$ do colo do útero, apontando para um aumento progressivo na prevalência de realização desse tipo de exame ${ }^{10,14-16}$. No cenário geográfico e conjuntural do presente estudo, notadamente onde não há registro de câncer, não foram encontradas pesquisas nessa temática, surgindo indagações referentes à adesão às diretrizes de rastreamento do CCU em uma dada realidade, ainda neste campo, inexplorada.

Dessa forma, o objetivo do presente estudo foi estimar a prevalência e identificar fatores associados ao $\mathrm{CP}$ do colo do útero em atraso (realizado há mais de três anos) entre mães com filhos menores de dois anos de idade em município da Região Sudeste do Brasil.

\section{Métodos}

Trata-se de um estudo de delineamento transversal realizado com mães de filhos menores de dois anos, residentes, em 2010, na Zona Norte do município de Juiz de Fora (MG). A pesquisa faz parte de um estudo mais amplo, conduzido pelo Núcleo de Assessoria, Treinamento e Estudo em Saúde (NATES) da Universidade Federal de Juiz de Fora (UFJF), intitulado "Inquérito de Saúde do Município de Juiz de Fora, MG”, cujo objetivo foi avaliar o acesso aos serviços de saúde materno-infantil.

O município de Juiz de Fora encontra-se localizado na microrregião da Zona da Mata do Estado de Minas de Gerais. Sua população, no ano de 2010, era de 516.247 habitantes, ocupando uma área de $1.429 \mathrm{~km}^{2}$. A região administrativa Norte concentra $23,6 \%$ da população do município em questão e possui a maior disponibilidade territorial na área urbana, além de possuir a maior concentração de crianças do município.

Os critérios de inclusão foram mulheres com filhos menores de dois anos de idade na data da entrevista, residentes na Zona Norte do município de Juiz de Fora (MG). O processo de seleção ocorreu por meio de amostragem aleatória complexa. As unidades primárias de amostragem foram os setores censitários, perfazendo um total de 22. Para o sorteio, os setores foram agrupados em estratos definidos de acordo com as diferentes modalidades de atenção à saúde às quais a população encontrava-se adstrita: Atenção Primária (com Estratégia de Saúde da Família ou atendimento tradicional), Atenção Secundária e Área Descoberta. A seleção dos setores foi feita com probabilidades proporcionais ao seu tamanho, de forma independente em cada estrato.

Para cálculo do tamanho da amostra de crianças com até dois anos de idade, considerou-se intervalo de confiança de $95 \%$ (IC95\%), correção para populações finitas, efeito do plano amostral igual a 1,5 e possíveis perdas por recusa como aproximadamente $15 \%$. A base populacional foi construída a partir de uma triagem (contagem rápida) realizada previamente ao início do estudo nos setores censitários urbanos pertencentes à área de abrangência da Zona Norte de Juiz de Fora selecionados para a amostra. O procedimento de triagem foi realizado por amostragem, onde um a cada cinco domicílios foi 
selecionado por amostragem sistemática com o objetivo de identificar a existência de residentes pertencentes ao grupo de interesse. Nos domicílios selecionados, buscou-se levantar informações referentes aos domicílios vizinhos (dois localizados à esquerda e dois localizados à direita).

Com base no método de triagem rápida por amostragem, estimaram-se 763 indivíduos de até 2 anos de idade. No entanto, no momento da entrevista, foram identificados, de fato, 497 pertencentes ao grupo de interesse. Além disso, as perdas somadas resultaram em um total de 152, incluindo mudanças de endereço ou quando a responsável pela criança não foi encontrada na casa, após serem realizadas 3 tentativas de contato em horários diferentes. Dessas perdas, as recusas corresponderam a 22 indivíduos. Ao final, foram aplicados 345 questionários. Desse total, 308 foram utilizados neste estudo, sendo excluídos 37, dos quais 9 questionários foram respondidos em duplicidade por se tratar de uma mesma mãe com 2 crianças menores de 2 anos. Os demais, totalizando 28, foram excluídos por não terem sido respondidos pela mãe bilógica.

Os dados foram obtidos por meio de um questionário estruturado em blocos temáticos, contendo questões referentes a situações demográficas e socioeconômicas da mulher, informações sobre o pré-natal, parto, puericultura, submissão ao $\mathrm{CP}$ do colo do útero, além de dados relacionados às características do domicílio e de seus moradores. Os entrevistadores foram estudantes provenientes da UFJF, que receberam treinamento para a realização das entrevistas. Os estudantes foram acompanhados, avaliados e orientados durante todo o período da pesquisa de campo. Para o controle de qualidade das informações coletadas, $10 \%$ da produção foi avaliada por nova entrevista parcial.

Optou-se pela escolha da variável dependente "exame citopatológico em atraso", sendo caracterizada como exame nunca realizado na vida ou, quando realizado, há mais de três anos antecedentes ao momento da entrevista. Para tanto, considerou-se a orientação do MS para a realização do $\mathrm{CP}$ do colo do útero anualmente e, após dois exames anuais consecutivos negativos, a repetição após três anos ${ }^{15}$.

Foram exploradas as associações entre o desfecho e algumas variáveis clássicas encontradas em estudos da mesma temática, disponíveis no questionário aplicado, agrupadas em três categorias. As variáveis foram: demográficas - idade (até 24 anos, de 25 a 35 anos, mais de 35 anos, considerando-se que a maior parte da amostra é constituída por mulheres jovens com filhos menores de 2 anos); cor da pele autorreferida (branca, preta, parda, morena, mulata, amarela e mestiça); estrato onde se encontrava o domicílio (com ou sem cobertura pela Estratégia de Saúde da Família); estado civil (solteira, casada, divorciada/viúva); número de gestações; número de filhos nascidos; indivíduo elegido como chefe da família; sexo e idade do chefe da família.
Entre as variáveis socioeconômicas, incluíram-se: saber ler e escrever; grau de escolaridade da mulher e do chefe da família (baixo - até o ensino fundamental; não baixo - ensino médio ou mais); número de moradores do domicílio; situação de emprego do chefe da família (não trabalha, empregado com carteira assinada, empregado sem carteira assinada, trabalha por conta própria, é patrão/empregador, trabalho não remunerado); renda pessoal e renda per capita da família; classificação socioeconômica segundo critérios da Associação Brasileira de Empresas de Pesquisa (ABEP); recebimento de benefício do governo (Bolsa Família, aposentadoria, auxílio doença, seguro-desemprego).

Quanto ao uso dos serviços de saúde, incluíram-se: acompanhamento pré-natal (sim ou não); local (Unidade Básica de Saúde - UBS, ambulatório de hospital, consultório particular, pronto atendimento, centro de especialidades e outros) e tipo de serviço para utilizado para o pré-natal (convênio, particular, Sistema Único de Saúde - SUS ou misto); mês de início do pré-natal; número de consultas de pré-natal; submissão ao CP durante o pré-natal (sim, não ou não sabe); local e tipo de serviço onde o último $\mathrm{CP}$ do colo do útero foi realizado; tempo de submissão ao último exame. Para as mulheres que nunca se submeteram ao exame, perguntou-se sobre os motivos relacionados a essa não realização.

Os dados primários resultantes da aplicação dos questionários foram codificados e digitados no programa Epi-Info 3.5.1, sendo posteriormente exportados, após críticas, revisões e correções de erros de digitação, para o pacote estatístico SPSS, versão 15.0, possibilitando a construção do banco de dados definitivo.

Inicialmente, a associação das variáveis independentes com o desfecho foi testada separadamente, utilizando-se o teste do $\chi^{2}$, observando-se aquelas que apresentaram um nível de significância menor ou igual a 0,05. A seguir, com o objetivo de medir a associação de uma variável independente, controlada pelas demais que mostraram associação com o desfecho na análise bivariada, realizou-se a análise multivariada por meio do modelo de regressão logística disponível no recurso complex sample do SPSS 15.0. Para tanto, as variáveis independentes foram inseridas no modelo agrupadas em blocos (demográficas, socioeconômicas e relacionadas aos serviços de saúde), considerando-se como critério para ordem de inserção as que apresentaram maior significância estatística na análise bivariada, possibilitando a construção de um modelo final.

Tanto para a análise bivariada quanto para a multivariada, utilizou-se um banco de dados ponderado que permitiu considerar o peso de cada unidade amostral, com objetivo de obter resultados que pudessem descrever de forma mais fidedigna as características da população estudada. 
O projeto de pesquisa foi aprovado pelo Comitê de Ética em Pesquisa com Seres Humanos da Universidade Federal de Juiz de Fora e obteve parecer favorável sob o $n^{\circ} 277 / 2009$, estando em conformidade com preceitos éticos que incluem o anonimato dos sujeitos participantes, identificados apenas por números, e a assinatura do termo de consentimento depois de informados claramente a respeito da pesquisa.

\section{Resultados}

Foram entrevistadas 308 mães de filhos menores de 2 anos. A amostra foi constituída por $45,7 \%$ de mulheres com idade entre 25 a 35 anos, com média de 27 anos, que possuíam em média 1,8 filhos nascidos e escolaridade predominante colegial completo ou superior incompleto $(40,6 \%)$. A maior parte das entrevistadas vivia com o companheiro $(75,7 \%)$ e informou ser casada (49,8\%). Em relação aos domicílios onde as mulheres residiam, verificou-se que $61,5 \%$ foram classificadas no estrato econômico $\mathrm{C}$, segundo a ABEP. A renda per capita domiciliar média foi de $\mathrm{R} \$ 284,00$.

O percentual de mulheres que se encontrava com o CP do colo do útero em atraso foi de $26,6 \%$ (IC95\% $21,3-32,6)$, incluindo-se aquelas que nunca se submeteram ao exame. Do total de mulheres submetidas ao CP alguma vez na vida, 6,7\% estavam com o exame em atraso (realizado há mais de 3 anos). Em relação ao local onde o último exame foi realizado, $43,8 \%$ destes foram feitos em
Unidade Básica de Saúde. Observou-se que 62,6\% dos atendimentos foram por meio do Sistema Único de Saúde.

A Tabela 1 mostra as características demográficas, socioeconômicas e relacionadas aos serviços de saúde para atendimento pré-natal das mulheres que estavam com $\mathrm{CP}$ do colo do útero desatualizado, comparadas às mulheres que estavam com a citologia atualizada. A maioria das mulheres com exame em atraso era mais jovem $(53,6 \%)$, considerava-se não branca $(63,7 \%)$, era solteira $(60,7 \%)$, possuía menor escolaridade $(72,2 \%)$ e pertencia às classes sociais baixas $(86,8 \%)$.

Todas as mulheres entrevistadas eram mães de filhos menores de dois anos; portanto, esperou-se inicialmente que a maioria delas informasse contato prévio com os serviços de saúde em virtude do pré-natal e puerpério. Observou-se que todas as mulheres que estavam com o CP do colo do útero em atraso foram atendidas no pré-natal. Dentre elas, 75,4\% iniciaram o acompanhamento pré-natal ainda no primeiro trimestre e $63,5 \%$ frequentaram de 7 a 10 consultas de pré-natal.

As mulheres que nunca haviam sido submetidas ao $\mathrm{CP}$ do colo do útero foram interrogadas em relação ao motivo dessa não submissão. As respostas mais frequentes foram a falta de conhecimento sobre o exame, no que se refere à finalidade e à importância, e o fato de julgarem não ser necessário realizá-lo por se sentirem saudáveis. Apenas $5,7 \%$ das entrevistadas referiram como motivo a dificuldade para marcar a consulta e realizar o exame.

Tabela 1. Características demográficas e socioeconômicas das mulheres que se encontravam com exame citopatológico em atraso

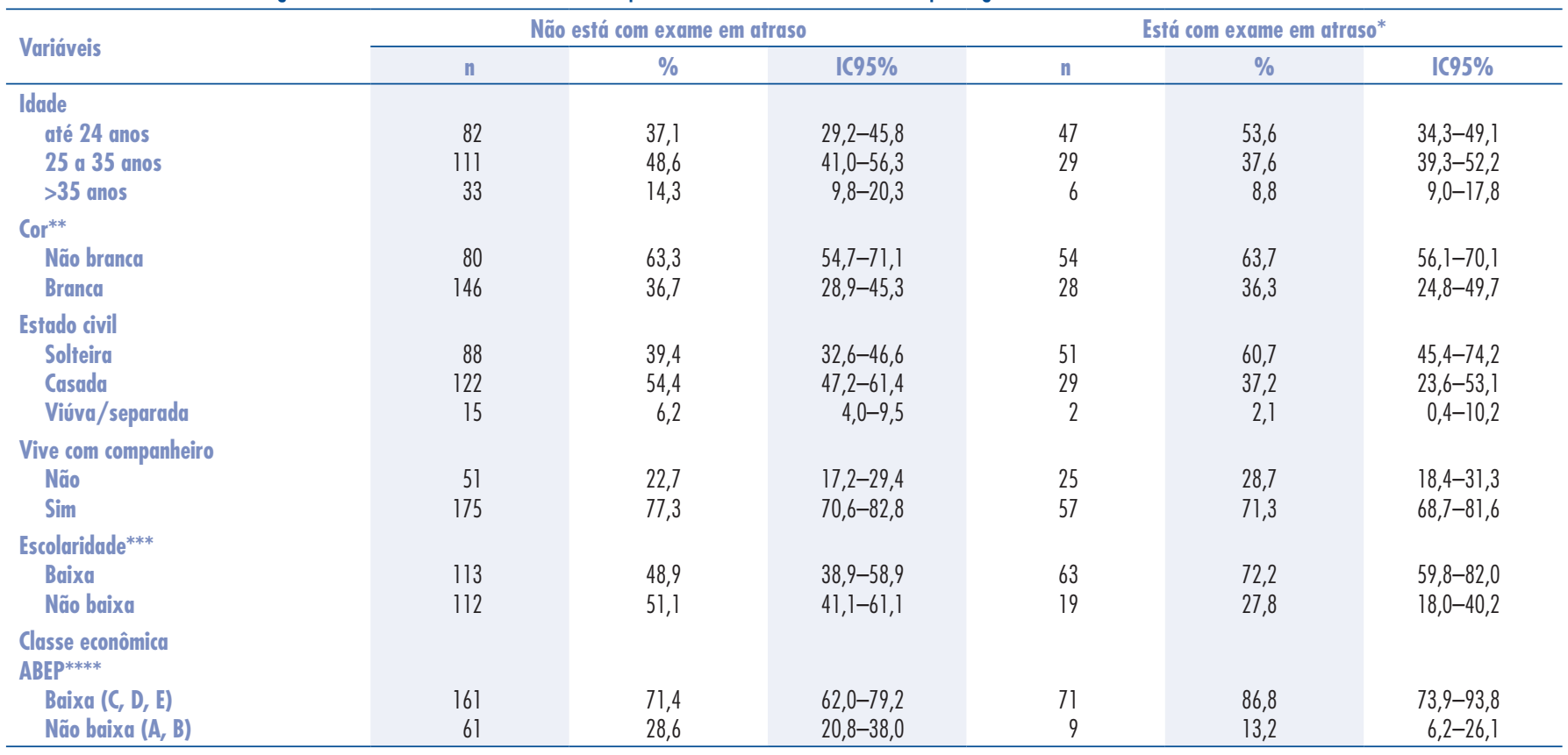

* exame considerado em atraso quando o último teste foi realizado há mais de três anos, incluindo as mulheres que nunca o realizaram; * * cor autorreferida pela entrevistada; *** escolaridade classificada como baixa (até o ensino fundamental) e não baixa (ensino médio ou mais); *** * estratos de consumo familiar da Associação Brasileira de Empresas de Pesquisa (ABEP). Os totais de n (226 para "não está em atraso" e 82 para "exame em atraso") e \% (100\%) nem sempre somam exatamente os valores totais, devido a alguns casos de respostas faltantes. 
A Tabela 2 apresenta a análise bivariada segundo variáveis demográficas, socioeconômicas e relacionadas aos serviços de saúde das mulheres entrevistadas. Verificou-se que oito variáveis apresentaram significância estatística ( $\leq \leq 0,05)$ com o desfecho: a idade e o estado civil; a

Tabela 2. Análise bivariada do desfecho exame citopatológico do colo do útero em atraso segundo variáveis demográficas, socioeconômicas e relacionadas aos serviços de saúde das mulheres

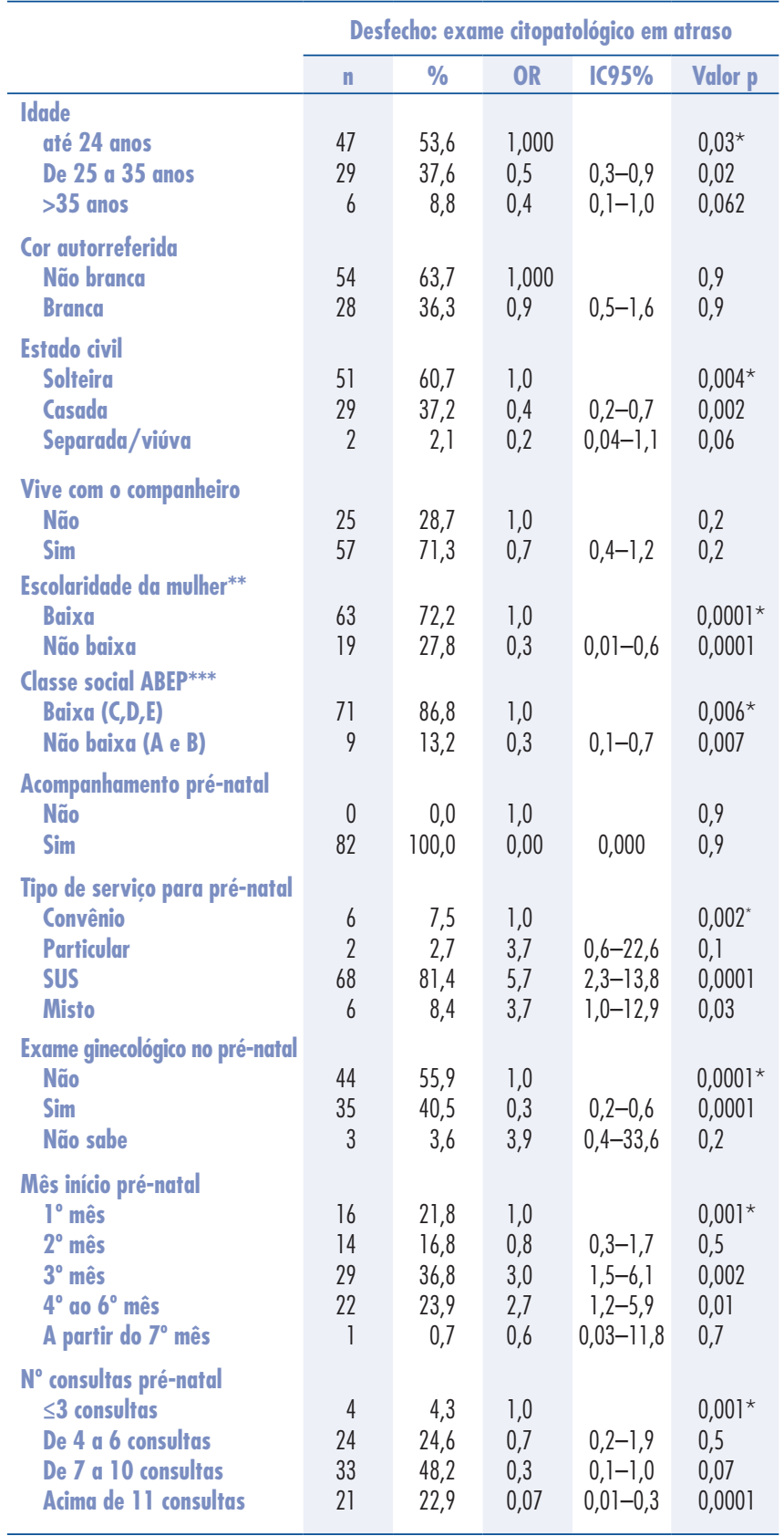

OR: razões de chances, estimadas pelo modelo de regressão logística para cada variável; * variáveis que apresentaram associação estatisticamente significativa com o desfecho; ** escolaridade baixa: ensino fundamental ou menos; escolaridade não baixa: ensino médio ou mais; *** estratos de consumo familiar da Associação Brasileira de Empresas de Pesquisa (ABEP); p: significância estatística do teste de associação do $\chi^{2} ; 1$ C95\%: intervalos de confiança para a estimativa pontual. Os totais de n (82) e \% (100\%) nem sempre somam exatamente os valores totais devido a alguns casos de respostas faltantes. escolaridade da mulher e a classe social; tipo de serviço utilizado para atendimento de pré-natal, submissão ao exame ginecológico durante o pré-natal, mês de início e número de consultas de pré-natal.

$\mathrm{Na}$ Tabela 3, encontra-se a análise multivariada com as variáveis independentes que mostraram associação significativa na análise bivariada. Para isso, mantiveram-se as variáveis demográficas, socioeconômicas e relacionadas aos serviços de saúde, sendo inseridas no modelo final, considerando-se a maior significância de associação obtida na análise bivariada. Ao final, observou-se que o estado civil casada e separada/viúva, ter se submetido ao exame

Tabela 3. Análise multivariada do desfecho exame citopatológico em atraso com as variáveis independentes de maior poder de associação na análise bivariada, utilizando-se o modelo de regressão logística, com as variáveis agrupadas em três blocos

\begin{tabular}{|c|c|c|c|}
\hline & \multicolumn{3}{|c|}{$\begin{array}{c}\text { Desfecho: exame citopatológico } \\
\text { em atraso }\end{array}$} \\
\hline & $\%$ & OR & IC95\% \\
\hline $\begin{array}{l}\text { Idade } \\
\text { até } 24 \text { anos } \\
\text { De } 25 \text { a } 35 \text { anos } \\
>35 \text { anos }\end{array}$ & $\begin{array}{r}53,6 \\
37,7 \\
8,7\end{array}$ & $\begin{array}{l}1,0 \\
0,7 \\
0,5\end{array}$ & $\begin{array}{l}0,3-1,4 \\
0,2-1,7\end{array}$ \\
\hline $\begin{array}{l}\text { Estado civil } \\
\text { Solteira } \\
\text { Casada } \\
\text { Separada/viúva }\end{array}$ & $\begin{array}{r}60,7 \\
37,2 \\
2,1\end{array}$ & $\begin{array}{l}1,0 \\
0,5^{\star} \\
0,1^{*}\end{array}$ & $\begin{array}{r}0,2-0,9 \\
0,02-0,8\end{array}$ \\
\hline $\begin{array}{l}\text { Escolaridade da mulher** } \\
\text { Baixa } \\
\text { Não baixa }\end{array}$ & $\begin{array}{l}72,2 \\
27,8\end{array}$ & $\begin{array}{l}1,0 \\
0,5\end{array}$ & $0,2-1,1$ \\
\hline $\begin{array}{l}\text { Classe social ABEP*** } \\
\text { Baixa }(C, D, E) \\
\text { Não baixa (A e B) }\end{array}$ & $\begin{array}{l}84,8 \\
12,9\end{array}$ & $\begin{array}{l}1,0 \\
0,5\end{array}$ & $0,2-1,2$ \\
\hline $\begin{array}{l}\text { Exame ginecológico no pré } \\
\text { Não } \\
\text { Sim } \\
\text { Não sabe }\end{array}$ & $\begin{array}{r}48,5 \\
47,0 \\
4,5\end{array}$ & $\begin{array}{l}1,0 \\
0,3^{*} \\
1,9\end{array}$ & $\begin{array}{r}1,0 \\
0,1-0,6 \\
0,1-28,7\end{array}$ \\
\hline $\begin{array}{l}\text { Tipo de serviço pré-natal } \\
\text { Convênio } \\
\text { Particular } \\
\text { SUS } \\
\text { Misto }\end{array}$ & $\begin{array}{r}7,5 \\
2,7 \\
81,4 \\
8,4\end{array}$ & $\begin{array}{l}1,0 \\
1,7 \\
2,9 \\
3,0\end{array}$ & $\begin{array}{r}0,2-14,5 \\
1,4-6,3 \\
1,0-9,4\end{array}$ \\
\hline $\begin{array}{l}\text { Mês de início do pré-natal } \\
1^{\circ} \text { mês } \\
2^{\circ} \text { mês } \\
3^{\circ} \text { mês } \\
4^{\circ} \text { ao } 6^{\circ} \text { mês } \\
\text { A partir do } 7^{\circ} \text { mês }\end{array}$ & $\begin{array}{r}21,8 \\
16,8 \\
36,8 \\
23,9 \\
0,7\end{array}$ & $\begin{array}{l}1,0 \\
0,5 \\
1,8 \\
1,1 \\
0,2\end{array}$ & $\begin{array}{r}0,2-1,2 \\
0,7-4,5 \\
0,4-3,3 \\
0,02-2,5\end{array}$ \\
\hline $\begin{array}{l}\text { Número de consultas pré- } \\
\leq 3 \text { consultas } \\
\text { De } 4 \text { a } 6 \text { consultas } \\
\text { De } 7 \text { a } 10 \text { consultas } \\
\text { Acima de } 11 \text { consultas }\end{array}$ & $\begin{array}{r}4,3 \\
24,6 \\
48,2 \\
22,9\end{array}$ & $\begin{array}{l}1,0 \\
0,7 \\
0,5 \\
0,09 *\end{array}$ & $\begin{array}{r}0,3-1,4 \\
0,2-1,2 \\
0,03-0,2\end{array}$ \\
\hline
\end{tabular}

OR: razões de chances, estimadas pelo modelo de regressão logística para cada variável; * variáveis que apresentaram associação estatisticamente significativa com o desfecho; **escolaridade baixa: ensino fundamental ou menos; escolaridade não baixa: ensino médio ou mais; ${ }^{* *}$ estratos de consumo familiar da Associação Brasileira de Empresas de Pesquisa (ABEP). IC95\%: intervalo de confiança para a estimativa pontual. Os totais de n (82) e \% (100\%) nem sempre somam exatamente os valores totais, devido a alguns casos de respostas faltantes. 
ginecológico durante o pré-natal e o número de consultas de pré-natal foram as variáveis que mantiveram associação significativa de forma positiva à submissão ao exame $\mathrm{CP}$ do colo do útero.

\section{Discussão}

Considerando-se o percentual de mulheres que estava com o CP em atraso, a prevalência do exame atualizado foi de $73,4 \%$, encontrando-se ligeiramente abaixo da cobertura de $80 \%$ recomendada pela Organização Mundial da Saúde (OMS) para causar impacto na incidência e na mortalidade por CCU. Resultados de coberturas superiores a $80 \%$ foram descritos em outras pesquisas realizadas em municípios brasileiros, como em São Leopoldo (RS), que apresentou cobertura de $80 \%{ }^{17}$, Florianópolis (SC), cuja cobertura encontrada foi de $93 \%^{14}$, Pelotas (RS), com cobertura de $86 \%^{15}$, e Rio Branco (AC), com 85,3\% de cobertura ${ }^{18}$. Em relação ao percentual de $\mathrm{CP}$ em atraso, incluindo-se as mulheres que nunca haviam sido submetidas ao exame anteriormente, cabe destacar que as pesquisas citadas incluem mulheres em diferentes faixas etárias, dificultando a comparabilidade dos resultados.

De certa forma, observa-se que a cobertura para o CP do colo do útero vem aumentando, ao mesmo tempo em que a incidência de CCU no país permanece elevada, sugerindo que o foco das ações de rastreamento está inadequado. $\mathrm{Na}$ realidade, as mulheres mais vulneráveis ao desenvolvimento do CCU são as que menos se submetem ao exame e, quando o fazem, não seguem a periodicidade recomendada pelo MS, gerando impacto direto no tratamento e prognóstico da doença ${ }^{4,10,11,19,20}$.

Em relação à idade, observou-se na análise bivariada que as mulheres com 25 a 35 anos, quando comparadas às mais jovens (até 24 anos), apresentaram menores chances de estarem com o CP em atraso. No entanto, a significância da associação do desfecho com a variável idade não se manteve na análise multivariada. Outros estudos, apesar de incluírem mulheres em diferentes faixas etárias, também encontraram proporções menores de realização do CP do colo do útero em mulheres mais jovens ${ }^{6,10,14}$.

Considerando-se os motivos citados nesta pesquisa e os encontrados em estudos com a mesma temática, observa-se que mulheres de menor idade são as que menos aderem a esse tipo de exame. Os principais motivos são: se sentirem saudáveis e suporem que não necessitam de cuidados de saúde, desconhecimento da necessidade do exame, vergonha, medo, constrangimento, ausência de problemas ginecológicos, entre outros ${ }^{10}$.
A variável estado civil mostrou associação significativa na análise multivariada para o grupo de mulheres casadas e para as mulheres separadas/viúvas, apontando que esses grupos apresentam menores chances de estar com o exame em atraso quando comparados ao grupo de mulheres solteiras. Em geral, estudos demonstram que as mulheres que vivem sem companheiro apresentam maior risco para o $\mathrm{CCU}^{15,16,18}$. Assim como este, outros estudos também demonstraram menor cobertura do exame entre as mulheres solteiras, encontrando maior cobertura entre as casadas, seguidas pelas separadas/ divorciadas. Essa situação foi interpretada considerando-se a prática sexual regular como mediadora para a realização do $\mathrm{CP}$, com consequente demanda aumentada por serviços de assistência ginecológica, devido à procura por serviços de planejamento familiar e obstétricos, oportunizando a realização do exame $\mathrm{e}^{10,18}$.

Em relação à variável classe social, verificou-se associação significativa na análise bivariada que não foi mantida na análise multivariada. Essa realidade sugeriu que a renda da população estudada é relativamente homogênea, já que a maioria das famílias foi classificada em classe social baixa (C, D e E). Cabe ressaltar que outras pesquisas na mesma temática encontraram associação significativa entre classe social e não submissão ao exame $\mathrm{CP}$ do colo do útero, cujos resultados apontaram para uma menor cobertura desse exame entre as mulheres que pertencem às classes sociais mais baixas e possuem menor renda familiar ${ }^{21-24}$.

A baixa escolaridade apresentou-se significativamente associada ao desfecho na análise bivariada, porém a significância da associação foi perdida na análise multivariada. Alguns estudos encontraram menor cobertura do exame entre as mulheres com menor escolaridade, destacando-se a baixa escolaridade como um fator de risco para o desenvolvimento do $\mathrm{CCU}^{10,11,15,20}$.

Considerando-se a utilização dos serviços de saúde entre as mulheres que se submeteram ao CP do colo do útero em algum momento da vida, observou-se que $51,7 \%$ dos exames foram realizados na Unidade Básica de Saúde e $62,6 \%$ dos atendimentos aconteceram no SUS. Apesar da maior proporção de exames feitos no SUS, os locais de realização do exame, bem como o tipo de serviço utilizado, não mostraram relação significativa com o fato de estar com a citologia desatualizada. Cabe ressaltar que alguns estudos encontraram relação significativa entre a situação de ser atendida por meio de planos de saúde e estar com a citologia atualizada, encontrando-se maior cobertura do CP entre mulheres que utilizam serviços privados de saúde ${ }^{20,21}$. 
Quanto ao tempo de realização do último CP do colo do útero, os achados desta pesquisa encontram-se consistentes com os demais estudos, nos quais a maioria das mulheres entrevistadas $(50,4 \%)$ informou ter se submetido ao último exame há um ano — ou menos da entrevista. Essa situação pode ser interpretada a partir da crença de muitas mulheres e profissionais de saúde de que a efetividade do exame aumenta com sua realização anual. A partir disso, muitas mulheres são rastreadas desnecessariamente, a intervalos curtos, implicando em aumento de custos para os serviços de saúde e dificultando o acesso de grupos mais vulneráveis que apresentam maiores chances de não submissão ao CP do colo do útero ${ }^{10,11}$.

Segundo a OMS e o MS, o período pré-natal e pós-parto representa uma excelente oportunidade para a prevenção do CCU, já que a realização do CP faz parte da rotina de pré-natal ${ }^{10,11}$. Considerando-se que todas as mulheres que se encontraram com o exame em atraso foram acompanhadas no pré-natal, deveriam, a princípio, estar com o mesmo atualizado, evidenciando uma lacuna na assistência realizada. Em relação ao número de atendimentos realizados durante o pré-natal, o estudo mostrou que quanto maior o número de consultas nesse período, menores são as chances de estar com o CP em atraso. Estudo semelhante realizado no município de Rio Grande (RS) evidenciou que um maior número de consultas de pré-natal favorece o contato com o serviço de saúde e, consequentemente, facilita a adesão ao exame de forma oportuna ${ }^{10}$.

Os resultados aqui demonstrados são consistentes com os encontrados nos estudos brasileiros de temática e delineamento semelhantes, mesmo considerando-se as diferentes abordagens metodológicas utilizadas.

No entanto, os resultados desta pesquisa devem ser interpretados com cautela, devido a algumas limitações que são inerentes aos estudos transversais: algumas mães podem ter esquecido ou omitido dados referentes à realização do $\mathrm{CP}$, podendo o autorrelato ser tendencioso, uma vez que ter se submetido ao exame é um comportamento esperado. Além disso, cabe destacar também que os relatos das entrevistadas com relação às práticas preventivas para CCU não foram checados quanto à veracidade em registros dos serviços de saúde.

Outra limitação deste estudo decorre da utilização de dados de um inquérito de saúde mais amplo, cujo objetivo não foi especificamente avaliar a realização do $\mathrm{CP}$ do colo do útero. É importante considerar que a coleta do CP faz parte do exame ginecológico. Portanto, é importante considerar que as mulheres entrevistadas podem ter dificuldade de diferenciar o exame ginecológico do procedimento de coleta do material para o CP. Apesar das limitações, a quase totalidade das pesquisas que tratam da mesma temática procedem desta maneira, ou seja, a partir das informações prestadas pelas mulheres entrevistadas.

\section{Referências}

1. Guerra MR, Gallo CV, Mendonça GA. Risco de câncer no Brasil: tendências e estudos epidemiológicos recentes. Rev Bras Cancerol. 2005; $51(3): 227-34$.

2. American Cancer Society. Cervix uteri. In: Global Cancer Facts \& Figures. 2nd ed. Atlanta: American Cancer Society; 2011 . p. 24-6.

3. Dows LS, Smith JS, Scarinci I, Flowers L, Parham G. The disparity of cervical cancer in diverse populations. Gynecol Oncol. 2008; 109(Suppl 2):S22-30.

4. Brasil. Ministério da Saúde. Instituto Nacional de Câncer. Coordenação Geral de Ações Estratégicas. Divisão de Apoio à Rede de Atenção Oncológica. Câncer do colo do útero. In: Estimativa 2012: incidência de câncer no Brasil. Rio de Janeiro: INCA; 2011 . p. 35-7.

5. World Health Organization. International Agency for Research on Cancer. Cervix cancer screening. Lyon: IARC Press; 2005. (IARC Handbooks of Cancer Prevention, 10).

6. Amorim VM, Barros MB, César CL, Carandina L, Goldbaum M. Fatores associados à não realização do exame de papanicolaou: um estudo de base populacional no Município de Campinas, São Paulo, Brasil. Cad Saúde Pública. 2006;22(1 1):2329-38.
7. Derchain SF, Longatto Filho A, Syrjanen KJ. Cervical intraepithelial neoplasia: diagnosis and treatment. Rev Bras Ginecol Obstet. 2005;27(7):425-33.

8. Albuquerque KM, Frias PG, Andrade CL, Aquino EM, Menezes G, Szwarcwald CL. Cobertura do teste de papanicolaou e fatores associados à não-realização: um olhar sobre o Programa de Prevenção do Câncer do Colo do Útero em Pernambuco, Brasil. Cad Saúde Pública. 2009;25(Suppl 2):S301-9.

9. Gonçalves CV, Dias-da-Costa JS, Duarte G, Marcolin AC, Garlet $G$, Sakai AF, et al. Exame clínico das mamas em consultas de pré-natal: análise da cobertura e de fatores associados em município do Rio Grande do Sul, Brasil. Cad Saúde Pública. 2008;24(8): 1783-90.

10. Brasil. Ministério da Saúde. Instituto Nacional de Câncer. Coordenação Geral de Ações Estratégicas. Divisão de Apoio à Rede de Atenção Oncológica. Diretrizes brasileiras para o rastreamento do câncer do colo do útero. Rio de Janeiro: INCA; 2011.

11. Brasil. Ministério da Saúde. Secretaria de Atenção à Saúde. Departamento de Atenção Básica. Atenção ao pré-natal de baixo risco. Brasília (DF): Ministério da Saúde; 2012. 
12. Cesar JA, Santos GB, Sutil AT, Cunha CF, Dumith SC. Pap smears among pregnant women in Southern Brazil: a representative crosssectional survey. Rev Bras Ginecol Obstet. 2012;34(1 1):518-23.

13. Olesen SC, Butterworth P, Jacomb P, Tait RJ. Personal factors influence use of cervical cancer screening services: epidemiological survey and linked administrative data address the limitations of previous research. BMC Health Serv Res. 2012;12:34.

14. Gasperin SI, Boing AF, Kupek E. Cobertura e fatores associados à realização do exame de detecção do câncer de colo de útero em área urbana no Sul do Brasil: estudo de base populacional. Cad Saúde Pública. 2011 ;27(7):1312-22.

15. Hackenhaar AA, Cesar JA, Domingues MR. Exame citopatológico de colo uterino em mulheres com idade entre 20 a 59 anos em Pelotas, RS: prevalência, foco e fatores associados à sua não realização. Rev Bras Epidemiol. 2006;9(1):103-11.

16. Oliveira MM, Silva AA, Brito LM, Coimbra LC. Cobertura e fatores associados à não realização do exame preventivo de Papanicolaou em São Luís, Maranhão. Rev Bras Epidemiol. 2006;9(3):325-34.

17. Muller DK, Dias-da-Costa JS, Luz AM, Olinto MT. Cobertura do exame citopatológico do colo do útero na cidade de São Leopoldo, Rio Grande do Sul, Brasil. Cad Saúde Pública. 2008;24(1 1):25 1 1-20.

18. Borges MF, Dotto LM, Koifman RJ, Cunha MA, Muniz PT. Prevalência do exame preventivo de câncer do colo do útero em Rio Branco,
Acre, Brasil, e fatores associados à não-realização do exame. Cad Saúde Pública. 2012;28(6):1156-66.

19. Ozawa C, Marcopito LF. Papanicolaou smear screening: coverage in two home surveys applied in the city of São Paulo in 1987 and 2001-2002. Rev Bras Gineol Obstet. 2011;33(5):238-45.

20. de Quadros CA, Victora CG, da Costa JS. Coverage and focus of a cervical cancer prevention program in southern Brazil. Rev Panam Salud Publica. 2004; 16(4):223-32.

21. Malta DC, Moura EC, Oliveira M, dos Santos FP. Usuários de planos de saúde: morbidade referida e uso de exames preventivos, por inquérito telefônico, Brasil, 2008. Cad Saúde Pública. $2011 ; 27(1): 57-66$.

22. Moraes JR, Guimarães PV, Paula FL, Ferreira ML, Guimarães RM, Luiz RR. Relação entre plano de saúde e a realização do exame Papanicolaou: uma aplicação de escore de propensão usando um inquérito amostral complexo. Rev Bras Epidemiol. $2011 ; 14(4): 589-97$

23. Neumann NA, Tanaka OY, Victora CG, Cesar JA. Qualidade e equidade da atenção ao pré-natal e ao parto em Criciúma, Santa Catarina, Sul do Brasil. Rev Bras Epidemiol. 2003;6(4):307-1 8.

24. Silveira DS, Santos IS, Costa JSD. Atenção pré-natal na rede básica: uma avaliação da estrutura e do processo. Cad Saúde Pública. $2001 ; 17(1): 131-9$. 